\title{
Reply to the Editorial
}

Deborah J. Ossip

Department of Public Health Sciences, University of Rochester Medical Center, Rochester, New York

The Associate Editor for this themed issue (Deborah Ossip) also wishes to express her appreciation on behalf of the Journal of Smoking Cessation to Dr Kenneth Ward for his outstanding job as Guest Editor to provide this compendium of lessons learned from LMICs around the globe regarding tobacco cessation research. 